\title{
CDF Run I $B$ Physics Results
}

\author{
Stephen Bailey ${ }^{1}$ \\ for the CDF Collaboration \\ Department of Physics, Harvard University, Cambridge, MA 02138, USA
}

\begin{abstract}
The CDF Run I $B$ physics program has been very successful, making numerous measurements over a wide variety of $B$ physics topics. Measurements have included masses and lifetimes; discovery of the $B_{c} ; B_{s} \rightarrow J / \psi \phi$ polarization; $B^{0} \leftrightarrow \bar{B}^{0}$ mixing; $\sin (2 \beta)$; and rare decay limits. Recent results include a search for $\Lambda_{b} \rightarrow \Lambda \gamma$ and a study of $B^{0} \rightarrow J / \psi K^{(*) 0} \pi^{+} \pi^{-}$decays. The tools and experience developed during Run I are quite valuable as CDF enters Run II.
\end{abstract}

PACS: 14.40.Nd; 13.25.Hw; 14.20.Mr

\footnotetext{
${ }^{1}$ bailey@fnal.gov
} 


\section{Introduction}

Over the past decade, the Collider Detector at Fermilab (CDF) collaboration has produced many precision $B$ physics measurements through its extremely successful $B$ physics program. Nearly $50 B$ physics papers have been published and several more are in process. The primary advantages of hadron machines for $B$ physics are twofold: a large $b$ quark production cross section combined with the ability to produce all forms of $b$ mesons and baryons. The primary disadvantage is that the cross section for generic QCD background is even larger than the $b$ production cross section. Over the course of Run I CDF capitalized upon the advantages and overcame the disadvantages to produce many precision measurements across a wide variety of $B$ physics topics. An overview of these results is presented in section 2, while section 3 focuses on a few recent results. Conclusions are given in section 4 .

\section{Overview of Results}

As mentioned in the introduction, the $b$ quark production cross section is quite large in hadronic collisions. CDF has measured the integrated production cross section for $B$ mesons with transverse momentum $p_{T}(B)>$ $6.0 \mathrm{GeV} / c$ and rapidity $|y|<1.0$ to be $2.54 \pm 0.22 \pm 0.53 \mu \mathrm{b}[1]$. This is orders of magnitude larger than the production cross section at $\Upsilon(4 S)$ or $Z^{0}$. The differential cross section is also consistently higher than QCD theory across the full $p_{T}(b)$ spectrum, as shown in figure 1 .

$\mathrm{CDF}$ has also made measurements of the masses and lifetimes of $B^{ \pm}$, $B^{0}, B_{s}, B_{c}$, and $\Lambda_{b}[2,3]$. In the case of $B^{ \pm}$and $B^{0}$, the CDF results contribute significantly to the world average while the $B_{s}$ and $B_{c}$ results and the $\Lambda_{b}$ mass measurement represent the world's best (or only) measurements of these quantities.

One of the strengths of CDF is the ability to study $B_{s}$ mesons. In addition to mass and lifetime measurements, Run I $B_{s}$ studies have set a $B_{s}$ mixing limit of $\Delta m_{s}>5.8 \mathrm{ps}^{-1}$ [4] and measured polarization in $B_{s} \rightarrow J / \psi \phi$ [7]. These measurements set the stage for CDF Run II $B_{s}$ studies which will include $B_{s}$ mixing, the $C P$-even $v s$. -odd lifetime difference, and $C P$ violation searches.

A highlight of the CDF Run I $B$ physics program was the measurement of $\sin (2 \beta)=0.79_{-0.44}^{+0.41}[5]$. This measurement used approximately $400 B^{0} \rightarrow$ 
$J / \psi K_{S}^{0}$ decays and three different $B$ flavor tagging methods. One of the flavor tagging algorithms, same-side tagging, was discovered at CDF [6] and will be a crucial tool for future measurements of $C P$ violation and $B_{s}$ mixing.

Table 1 shows the CDF Run I rare decay limits [8]. $\operatorname{BR}\left(B^{0} \rightarrow \mu^{+} \mu^{-} K^{* 0}\right)<$ $4.0 \times 10^{-6}$ is especially promising since it is near the Standard Model prediction of $(1.0-1.5) \times 10^{-6}$.

\section{Recent Results}

\subsection{Search for $\Lambda_{b} \rightarrow \Lambda \gamma$}

A recent study performed the first search for baryonic electroweak penguin decays in $\Lambda_{b} \rightarrow \Lambda \gamma$. Like other electroweak penguin decays, this mode is sensitive to new physics contributions, both in the decay rate and in the polarization of the $\Lambda$ in its rest frame with respect to the direction of the $\Lambda$ momentum in the $\Lambda_{b}$ rest frame [9].

The search looked for $\Lambda \rightarrow \mathrm{p} \pi$ and $\gamma \rightarrow e^{+} e^{-}$using $\gamma$ conversions in the inner detector. The cuts optimized $\epsilon_{s i g} / \sqrt{\epsilon_{b k g}}$ where $\epsilon_{s i g}$ and $\epsilon_{b k g}$ are the signal and background efficiencies, respectively. The signal efficiency was determined using a Monte Carlo simulation while the backgrounds were modeled using the events in the sidebands of the $\Lambda_{b}$ mass plot. The mode $B^{+} \rightarrow J / \psi\left(\rightarrow e^{+} e^{-}\right) K^{+}$was used for efficiency normalization for determining the branching ratio limit. The results are shown in figure 2 with two events in the cross hatched signal region with an expected background of $3.4 \pm 0.6$ events (top) compared with $24.0 \pm 5.3$ events in the reference mode signal region (bottom). After considering the various efficiencies for the signal and reference modes and their uncertainties, these results correspond to a $90 \%$ confidence level branching ratio limit of

$$
\operatorname{BR}\left(\Lambda_{b} \rightarrow \Lambda \gamma\right)<6.5 \times 10^{-4}
$$

In comparison, Standard Model predictions are in the range $0.2-5 \times 10^{-5}$ [9].

\subsection{Study of $B^{0} \rightarrow J / \psi K_{S}^{0} \pi^{+} \pi^{-}$}

Another recent study has searched for $B^{0} \rightarrow J / \psi K_{S}^{0} \pi^{+} \pi^{-}$decays with a particular interest in the $B^{0} \rightarrow J / \psi K_{S}^{0} \rho^{0}$ contribution. Like $J / \psi K_{S}^{0}$, both 
$B^{0}$ and $\bar{B}^{0}$ can decay to this final state making this mode sensitive to $C P$ violation due to mixing. Unlike $J / \psi K_{S}^{0}$, however, $J / \psi K_{S}^{0} \rho^{0}$ is not a $C P$ eigenstate, thus it allows interference terms between the $C P$-even and $C P$ odd contributions. These cross terms are proportional to $\cos (2 \beta)$, a quantity whose measurement (or even sign) is useful for resolving the four-fold discreet ambiguities on $\beta$ from a $\sin (2 \beta)$ measurement.

The search cuts were optimized using $B^{0} \rightarrow J / \psi K_{S}^{0}$ as a reference signal. The resulting mass peak is shown at the top of figure 3 with $15.7 \pm 4.3$ events. The bottom plot in figure 3 shows the $B^{0} \rightarrow J / \psi K_{S}^{0} \rho^{0}$ candidate events remaining after $B^{0} \rightarrow J / \psi K^{*+} \pi^{-}$candidates have been removed. With such limited statistics, it is difficult to uniquely identify the $\rho^{0} \rightarrow \pi^{+} \pi^{-}$ contribution, but the events within the $B^{0}$ mass region fall within the $\rho^{0}$ spread and do not extend as wide as would be expected from non-resonant $\pi^{+} \pi^{-}$or other possible decay contributions such as $B^{0} \rightarrow J / \psi K_{0}^{*}(1430)^{+} \pi^{-}$. If these events are due to $B^{0} \rightarrow J / \psi K_{S}^{0} \rho^{0}$ and there is no interference with the $B^{0} \rightarrow J / \psi K^{*+} \pi^{-}$contribution, this corresponds to a branching ratio of:

$$
\mathrm{BR}\left(B^{0} \rightarrow J / \psi K_{S}^{0} \rho^{0}\right)=\left(5.5 \pm 2.5_{-1.1}^{+1.0}\right) \times 10^{-4} .
$$

The $K_{S}^{0} \pi^{+} \pi^{-}$mass plot indicates that some, but not all, of the signal events come from $B^{0} \rightarrow J / \psi K_{1}(1270)$.

\subsection{Other}

The analyses presented here are just a selection of several recent Run I analyses. Other ongoing analyses include $B_{s}$ mixing limits and measurements of $\mathrm{BR}\left(B^{+} \rightarrow J / \psi K^{+} \pi^{+} \pi^{-}\right)=(1.2 \pm 0.3 \pm 0.2) \times 10^{-3} ; \mathrm{BR}\left(B^{+} \rightarrow \chi_{c} K^{+}\right)=$ $(10.4 \pm 3.6 \pm 1.6) \times 10^{-4} ; \mathrm{BR}\left(B^{0} \rightarrow J / \psi K^{* 0} \pi^{+} \pi^{-}\right)=\left(5.3 \pm 1.1_{-0.9}^{+0.8}\right) \times 10^{-4}$; $\mathrm{BR}\left(B_{s} \rightarrow J / \psi \bar{K}^{* 0}\right)<8.5 \times 10^{-5}$; and a search for $B_{s} \rightarrow J / \psi K^{(*) 0} \bar{K}^{(*) 0}$.

\section{Conclusions}

Much has been accomplished in the CDF Run I $B$ physics program. We have capitalized upon the advantages of the hadronic environment while overcoming the primary disadvantage of large QCD backgrounds. In addition to demonstrating precision $B$ physics measurements at a hadronic accelerator, we have developed tools and experience which are crucial as we enter Run II. We expect many new results in Run II while receiving stiff competition 
from the $e^{+} e^{-} B$ factories. All of this has been accomplished at CDF using what is in essense a general purpose high energy particle detector. When CDF Run II ends, we look forward to a strong continuation of $B$ physics in the hadronic environment at the dedicated $B$ physics experiments of $B \mathrm{TeV}$ and LHCb. It is an exciting time for $B$ physics and hadronic machines are a great place to be.

\section{Acknowledgements}

I would like to thank Yoram, Liz, and the other conference organizers for a great physics and social program. I would also like to thank Gil, Hagar, Simon, Gildad, Liz, Shulamit, and others who helped me plan my postconference walk to Jerusalem (I made it!).

\section{References}

[1] F. Abe et al., Phys. Rev. D 60, 092005 (1999)

[2] F. Abe et al., Phys. Rev. D53, 3496 (1996), Phys. Rev. Lett. 81, 2432 (1998), Phys. Rev. D55, 1142 (1997)

[3] CDF $b$ meson and baryon lifetime measurements are summarized at: http://www-cdf.fnal.gov/physics/new/bottom/blife_summary/blife_summary.html

[4] F. Abe et al., Phys. Rev. Lett. 82, 3576 (1999)

[5] T. Affolder et al., Phys. Rev. D61, 072005 (2000)

[6] F. Abe et al., Phys. Rev. Lett. 80, 2057 (1998)

[7] T. Affolder et al., Phys. Rev. Lett. 85:4668-4673 (2000)

[8] T. Speer et al., DPF 2000 Proceedings, pub: World Scientific (2000)

T. Affolder et al., Phys. Rev. Lett. 83, 3378 (1999)

F. Abe et al., Phys. Rev. D57, R3811 (1998)

[9] T. Mannel and S. Recksiegel, J. Phys. G24, 979 (1998)

C. Huang and H. Yan, Phys. Rev. D59, 114022 (1999)

R. Mohanta et al., hep-ph/9908291 (1999) 


\begin{tabular}{ll} 
Mode & BR Limit $(90 \%$ CL) \\
\hline$B^{0} \rightarrow K^{* 0} \gamma$ & $1.1 \times 10^{-4}$ \\
$B_{s} \rightarrow \phi \gamma$ & $1.2 \times 10^{-4}$ \\
$B_{s} \rightarrow \mu^{+} \mu^{-} \phi$ & $4.2 \times 10^{-5}$ \\
$B^{+} \rightarrow \mu^{+} \mu^{-} K^{+}$ & $5.2 \times 10^{-6}$ \\
$B^{0} \rightarrow \mu^{+} \mu^{-} K^{* 0}$ & $4.0 \times 10^{-6}$ \\
$B^{0} \rightarrow \mu^{+} \mu^{-}$ & $8.6 \times 10^{-7}$ \\
$B_{s} \rightarrow \mu^{+} \mu^{-}$ & $2.6 \times 10^{-6}$
\end{tabular}

Table 1: CDF rare $B$ decay limits. 


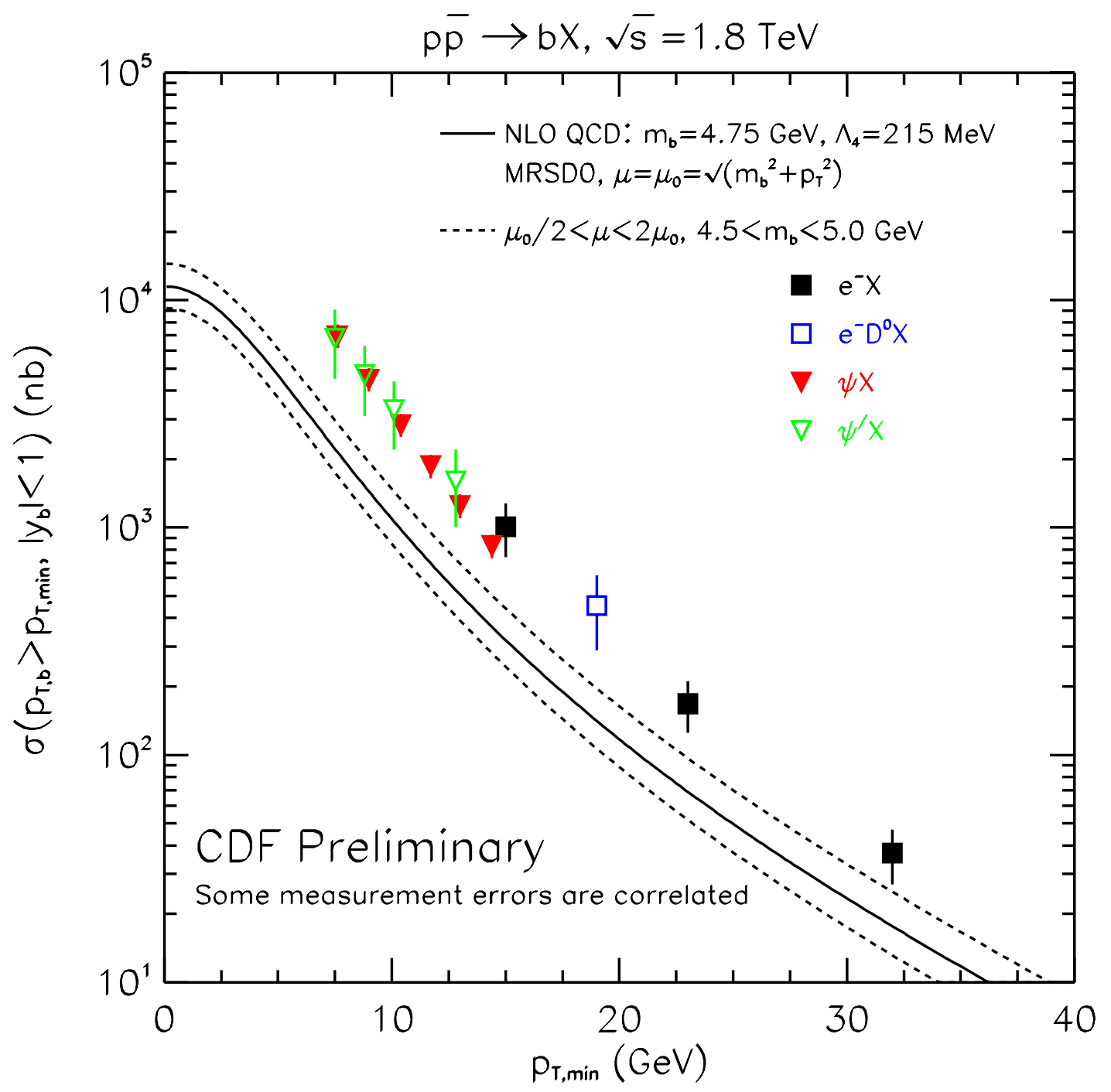

Figure 1: $b$ differential production cross section at $\sqrt{s}=1.8 \mathrm{TeV}$. 


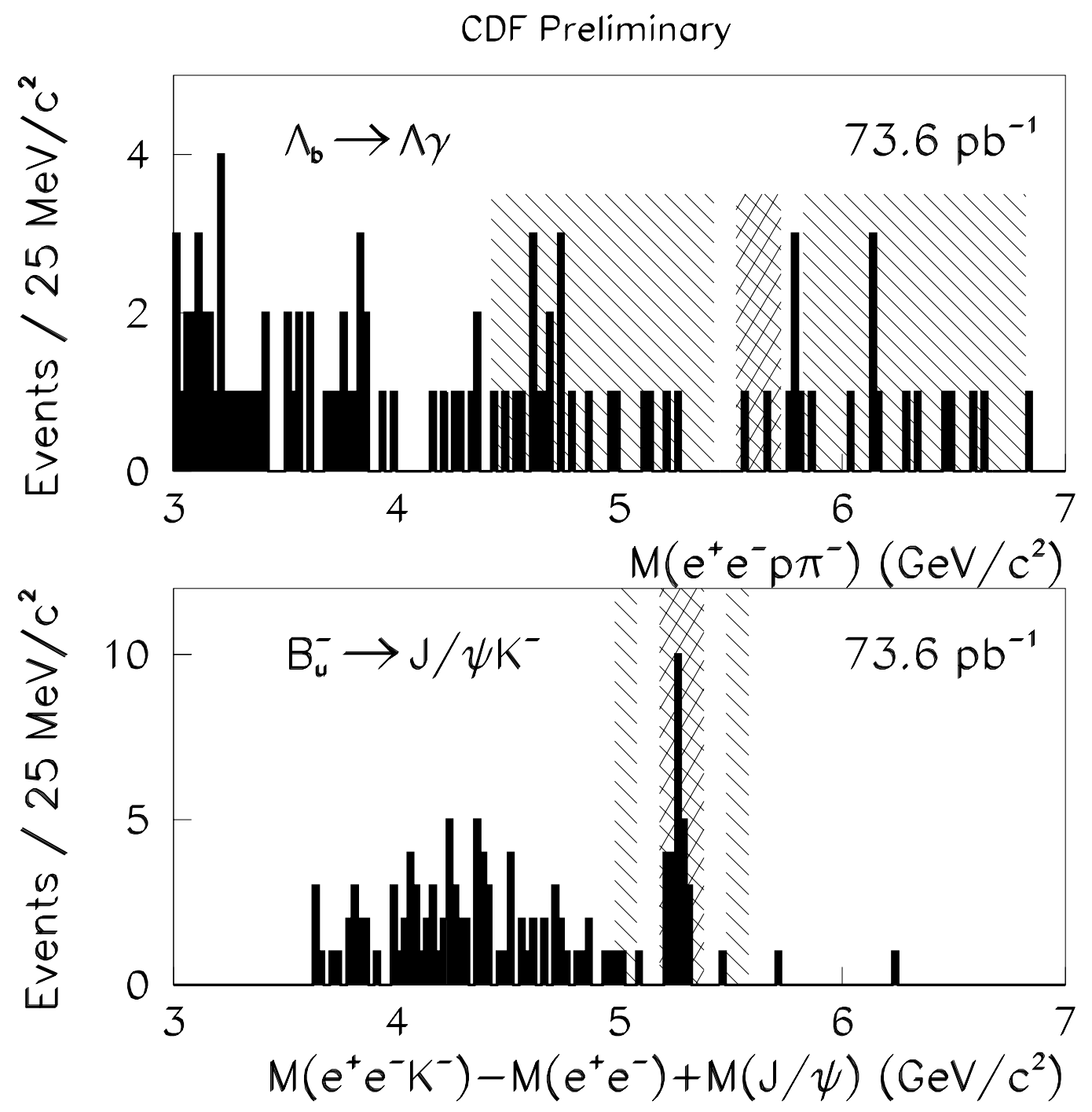

Figure 2: $\Lambda_{b} \rightarrow \Lambda \gamma$ candidates (top) compared to the $B^{-} \rightarrow J / \psi(\rightarrow$ $\left.e^{+} e^{-}\right) K^{-}$reference signal (bottom). 

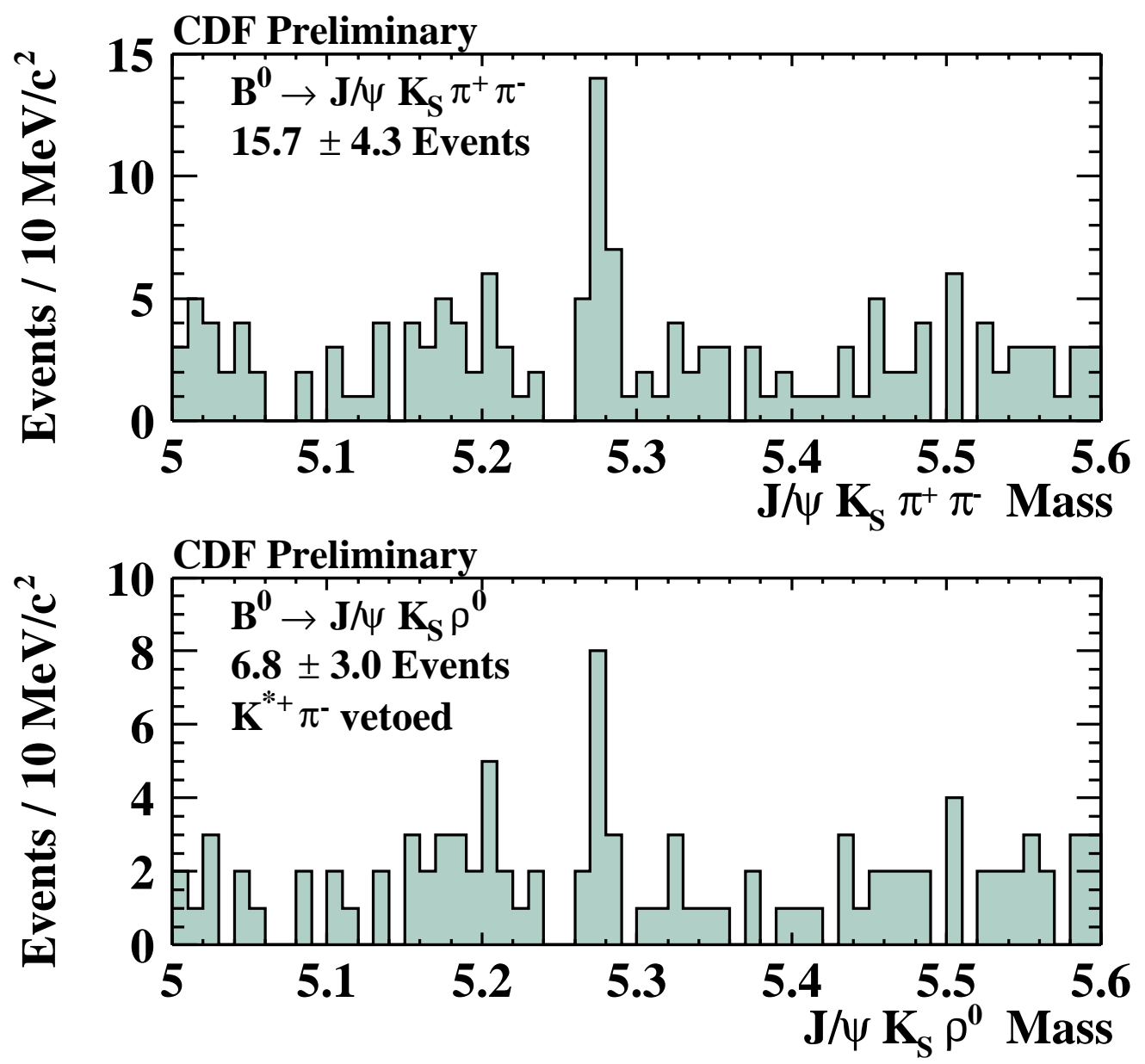

Figure 3: $B^{0} \rightarrow J / \psi K_{S}^{0} \pi^{+} \pi^{-}$candidate mass peak (top) with $15.7 \pm 4.3$ events. The bottom plot shows the $B^{0} \rightarrow J / \psi K_{S}^{0} \rho^{0}$ events remaining after $B^{0} \rightarrow J / \psi K^{*+} \pi^{-}$candidates have been removed. 